\title{
The Development of Qingdao Piano Art in Shandong Province (1940-1966)

\author{
Qing $\mathrm{Yu}$
}

Conservatory of Music, Qingdao University, Qingdao, China, 266071

artyuq@163.com

Keywords: Shandong, Qingdao, keyboard, piano, music, education

\begin{abstract}
Qingdao City, located in Shandong Province, has a strong piano music atmosphere, known as the "Qin Island". This is related to the unique culture which fuses both Chinese and Western culture in Qingdao history. This paper focuses on the teaching history of Chinese and foreign piano teachers in Qingdao from 1940 to 1966 and the keyboard music course development in Qingdao Normal School. It has trained several generations of music teachers and piano players in Qingdao therefore promote the development of Qingdao piano music.
\end{abstract}

\section{Introduction}

Qingdao, as a harbor trade city, the colonialist invasion also brought a religious culture. At the beginning of the twentieth century, some western missionaries founded churches and missionary schools in Qingdao, thus a group of people were converted to Christianity. They learnt to sing hymns, play the organ and piano from missionaries and nuns. In the thirties of the twentieth century, piano teaching activities in Qingdao mainly engaged in music classes and piano elective courses in missionary schools. These schools hired Chinese and foreign professional piano teachers, for example, the Shenggong female school (Qingdao No. seventh middle school) hired American nun Felders and Swiss Mrs. Reid; Wende female school hired teacher Yulan He and Qijing Tang who graduated from Shanghai State Conservatory of Music [1]. They engaged in both professional and amateur piano teaching activities. Moreover, they guided and lead more people to be engaged in music education after the founding of PRC. In addition, these schools organized a variety of performances including piano solo and accompaniment, which promoted the piano in the popularity in Qingdao.

In the early 1940s, due to the instability of the world situation, the foreign piano teachers have left Qingdao; however, there are many aliens' families to evacuate to Qingdao including many doctors of music in piano and professional piano teachers such as Austrian Dr. Karehalish, Russian Richard, South Korean Jinghe Xu, Sung-Pak Park and so on. There are also many influential piano teachers at the same period such like Pastor Jiting Dong, Yuezhen Dai. Moreover, Chongsheng Wang, Weifang Teng, Aiyu Wang and Ziyi Jia who was studying abroad in South Korea and the United States [2]. Some of these teachers had been taught in Qingdao Sili missionary school and Zhenguang female school which was founded by Presbyterian Church in America. They held early piano solo and ensemble concert in the history of Qingdao. Furthermore, they were piano accompaniment for various music classes and concerts, which made great contributions to the development of piano music in Qingdao and cultivate talents. For example, in 1950s, Lingfen Wu was admitted to Central Conservatory of Music in music composition major; Bingzhen Wang, Ruiqing Su, and Meiyu Zhou were also admitted in piano major. Wenju Sui was admitted to Wuhan Conservatory of Music in the same period. There were more pianists in 1960s such like Shiguang Cui and Keli Xu in Central Conservatory of Music; Zujin Chen and Houli Li in Tianjin Conservatory of Music. 


\section{Chinese and Foreign Piano Teachers}

\subsection{Dr. Heinrich}

Dr. Heinrich is an Austrian piano doctor, he taught piano in Wende female school voluntarily from 1940 to 1946. He had superb piano playing skills, various composition, and systematic piano teaching method with rigorous attitude, which attracted many students. They learnt classic works and different playing style of various periods in Europe. He made great contributions to cultivating piano talents in Qingdao such like Yuan Mao who became famous Chinese composer [3] and teachers like Ziyi Jia, Xiuwen Li, and so on. Figure 1 shows a photo by Dr. Heinrich and some students. Composer Yuan Mao is in the second position from right in front row.

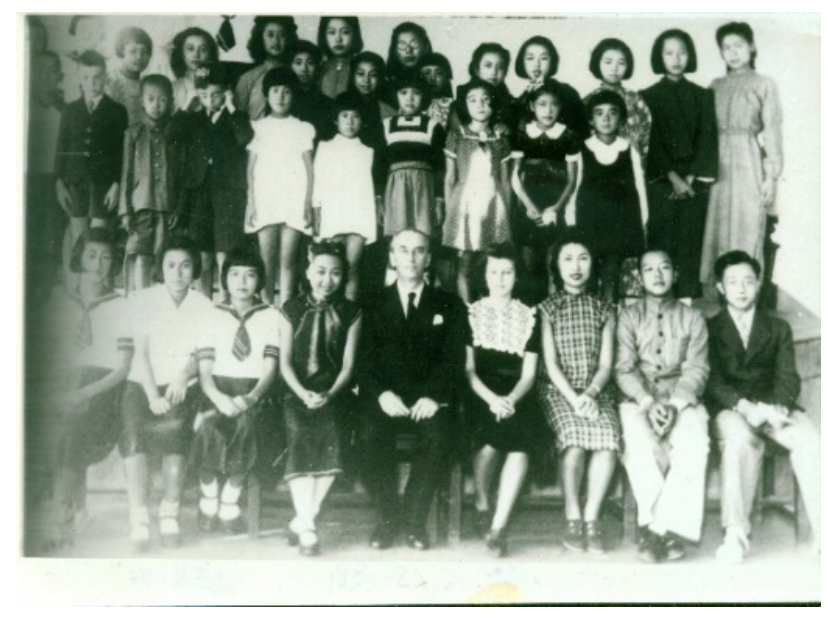

Fig.1. A Photo of Dr. Heinrich and Students

\subsection{Jinghe $X u$}

Korean Jinghe Xu (1911-2003), who is a piano teacher graduated from the music department of Ewha Womans University. She followed her husband Pastor Zhiri Fang to do missionary work in China from April of 1937, and then they moved their Christian church from Laiyang to Qingdao in Shandong province due to the Japanese aggression war in 1938. In the church, Jinghe Xu and her daughter played piano accompaniment for hymns and organized the choir for holy music [4]. Usually, she taught students at home and played the works of the European classical period. She focused on learning and researching modern teaching methods. Moreover, her teaching was patient, meticulous, and strict especially, which leads to pretty playing skills of her students. She had a number of students, for example, Guangxia Wang, Yumei Wang, Chongsheng Wang, Wenju Sui, Xiaoting Li, Tianmin Wang, and so on. Most of them were admitted to conservatory of music and became piano educator, therefore, made contributions to the development of piano music in Qingdao. Jinghe $\mathrm{Xu}$ and her husband stayed in Qingdao for nearly 20 years and came back to their homeland in 1957.

\subsection{Yuezhen Dai}

Yuezhen Dai (1909-1995), was born in Qingdao and taught by foreign piano teachers in Wende female school. During the years there, her playing skills improved rapidly. After the graduation, she studied piano in the University of Shanghai for two years. Then she came back to Qingdao and taught piano at the missionary school and Qingdao municipal female school. Her career as a piano teacher lasted more than fifty years, from 1930s to 1990s. She and her students held multiple concerts after the founding of PRC. In 1953, she presented the "Hungarian rhapsodies" and "La campanella" of Liszt, which attracted pretty good social response. She trained many a group of piano talent, such as Ciliang Sui in 1940s, Wenju Sui, Yumei Ding, Duosi Chang, Hanqi Wei and Bixuan Guo in 1950s. Furthermore, some of her students had been admitted to the National 
Academy of Music, Central Conservatory of Music, and other colleges. They became the local and national core group to promote the development of art in those years.

\subsection{Chongsheng Wang}

Chongsheng Wang (1918-2001)'s ancestral home ws in Changle and her parents were both devout Christians. Her father, Xuanchen Wang, was an outstanding national industry pioneer and devotes his whole life to service church and education. Her mother, Bingxian Meng, was born in Weixian and played piano accompaniment for hymns. Chongsheng Wang started study piano from her mother in five years old and she was in church school which help her improving English and music skills. In 1936, she began to study piano in the music department of Ewha Womans University and in 1937 she was admitted to the University of Marymans in Ohio State, USA. She graduated from the college and obtains the bachelor's degree in 1941. Her graduation concert received a good evaluation. When she came back to Qingdao, her father held a well-established solo and ensemble concert in Qingdao concert hall where in Lanshan road. Chongsheng Wang played the Beethoven's first piano concerto and achieved great response.

In 1942, Chongsheng Wang taught English and music in Wende female school and Shenggong female school. And she played piano accompaniment for hymns on Sunday in Christ church. She and her students held multiple concerts in various concert halls. Beside the working, she focused on piano education for nearly 60 years and cultivate thousands of piano talents. Her students were admitted to professional conservatory of music and some of them achieved doctor of music in piano. Chongsheng Wang occupies an important position in development history of piano in Qingdao.

\section{The Establishment of Piano Music Education in Qingdao}

After the founding of PRC, the earliest established music education institution in Qingdao is Licun normal school which is founded in 1930 and it is originally named Qingdao municipal country normal school. The name had been changed to Shandong Qingdao Normal school since 1950. It has the earliest music course in Qingdao and the school running has been expanding. Aiyu Wang and her husband Zheng Li started teaching music since 1951 in the school. There are special music courses for organ and piano. They cultivated a number of music teachers for elementary and middle schools, which promotes the development of piano and music.

\subsection{Aiyu Wang}

Aiyu Wang was born in Beijing in 1918, and began studying piano from 7 years old. Then her mother moved her family to Yantai and Aiyu Wang went to Zhenguang female school which was founded by Christian church. The piano teacher in the school is Dr. May. Aiyu Wang studied piano playing from Dr. May for 8 years, from the age of 10 to her graduation from kindergarten training school. The piano training of Dr. May was standard and systematic, and focused on basic skill training especially. That helped Aiyu Wang improving her playing skills and she was one of the best students in the school. After graduated from school, she became a music teacher in kindergarten and Qingdao Shenggong elementary school. She has also studied piano playing from a nun in Shenggong middle school to improve skills. In 1940, Aiyu Wang applied and got the music teacher position in Qingdao municipal female school (Qingdao No. 2 High School), and worked there for nearly 40 years.

\subsection{The Beginning of Keyboard Music Teaching}

In 1951, the Education Secretary persuaded Aiyu Wang and her husband Zheng Li to work in the Qingdao Normal School to cultivate teachers for elementary school. The school was remote and needy at that time and their child was very young, even though, they complied with the arrangement and worked there until 1962. At that time, the teaching condition was simple and crude. The only couple pianos and organs are old and shabby and there was no specified room. Therefore, they built 12 piano rooms with students. The rooms were very small that can only place one piano and a chair. 
After years' working, more than 40 piano rooms were built and every student in the school learnt organ. The organ class became required course in the course plan.

Moreover, Aiyu Wang opened the individual organ class for students which improved their skills rapidly. Since the students were very young and had no experience, Aiyu Wang taught them patiently and helped them. This is the beginning of keyboard course in Qingdao history.

\subsection{Self-compiled Testbooks}

At that year, there was no material for music teaching in Qingdao Normal School. Therefore, Aiyu Wang and Zheng Li compiled various textbooks for students in the spare time, such as "Organ textbook", "Sightsinging textbook", "Song anthology", and so on. Furthermore, they also compiled textbook to help students becoming excellent teacher in elementary school. These works improved the music teaching plan for multiple grades.

\subsection{Making Teaching Aids}

During the period of Great Leap Forward in 1957, the country was engaged in innovation. Aiyu Wang and Zheng Li created and produced a powered teaching aid with sound and lighting to help music teaching. It was demonstrated in the teaching expo held by Qingdao education bureau. The aid was a powered panel which can be hung on the top of piano. There was musical notation on the panel and marked with syllables, enharmonic notes and so on. The keyboard had a diatonic scale labeled by numbered musical notation which can be moved horizontally. It could show the correspondence between phoneme and interval in different tones. The panel could generate sound and light effects when keyboard was touched, which helped students to read music. With the help of this aid, many students could practice sightsinging and study music theory together. It increased the teaching efficiency and effect.

\subsection{Setup Hobby Groups}

In addition to teaching, Aiyu Wang and Zheng Li set up student hobby group including instrumental music, dancing group, compilation group, and vocal group. Beside the rehearsal of group dancing, they helped students building instruments workshop. The compilation group had created plenty of musical works with the guidance of teachers, such as dance drama "Shengjiao Luo", "Wenxue Liu”, "Fisherman’s Song in Baisha”, and chorus “Afforestation and Water Conservancy". Moreover, the teachers often organized students to the military tour and Aiyu Wang presented piano solo "Shepherd Boy’s Piccolo" and "Evening Party” which are composed by Luting He. Also, she played piano accompaniment for vocal solo and chorus.

\subsection{Piano Amateur Teaching}

In the spare time, Aiyu Wang also engaged in piano amateur teaching; cultivate a lot of art talent, and some students admitted to the institutions of higher learning. And many students became teachers in secondary schools.

\section{Keyboard Music Research}

In the piano music development history, the research on keyboard accompaniment and solo playing is relatively few. Huaxuan Li had done the research on keyboard music, who was a famous musician, educator, and a teacher in Qingdao No. 1 high school. He published the research paper “A discussion of coordinating keyboard instruments with national music” in 1963.

\section{Conclusion}

Through the collection of information, interviews with future generations, we have researched the development history of keyboard music in Qingdao from 1940 to 1966. It is shown that there were three stages in the development. The first stage is that foreign teachers of missionary schools 
started piano teaching, which promoted the popularity of piano in Qingdao. The second stage is that many high level piano teachers came to Qingdao and improved students' skills rapidly, such as Dr. Heinrich and Jinghe $\mathrm{Xu}$. They brought advanced teaching philosophy and methods. The third stage is that Qingdao normal school set keyboard courses and cultivated many music teachers for elementary and secondary schools. Moreover, many piano teachers like Yuezhen Dai, Chongsheng Dai and Aiyu Wang studied piano from foreign teachers and studied abroad. They cultivated many ourstanding students and accelerated the development of piano music in Qingdao.

\section{References}

[1] Department of Culture History Office of the Chinese Musicians Association of Shandong Branch, "Art information and music album" 1990.

[2] Huang Wen master's degree thesis "Qingdao piano art development course" 2010.

[3] Qu Jinliang , "Blue Qingdao" Qingdao Publishing House, June 2012.

[4] Wang Jinsheng, "Art Qingdao" Qingdao Publishing House, May 2012. 